\title{
O trabalho em saúde mental: um estudo de satisfação e impacto
}

\author{
Work in mental health: a job satisfaction and work \\ impact study
}

\footnotetext{
${ }^{1}$ Instituto Municipal Juliano Moreira, Rio de Janeiro, Brasil.

2 Instituto de Estudos de Saúde Coletiva, Universidade Federal do Rio de Janeiro, Rio de Janeiro, Brasil.

Correspondência D. Rebouças Instituto Municipal Juliano Moreira.

Rua Professor Hermes de Lima 511, apto. 301,

Recreio dos Bandeirantes, $R J$ 22795-065, Brasil. denisereboucas@globo.com
}

\section{Abstract}

Knowledge of job satisfaction and work impact among psychiatric staff is highly useful for policymakers and mental health professionals. Since there are few studies on this issue in Brazil, a cross-sectional study was carried out among mental health professionals. Data were collected for 133 professionals from 4 mental health services in Rio de Janeiro, using SATIS-BR and IMPACTO-BR scales and a socio-demographic questionnaire. Statistical associations were analyzed using the Mann-Whitney, Kruskal-Wallis, and chi-square tests and multiple linear regression. SPSS 10.1 for Windows was used for statistical analyses. Mean satisfaction was 3.30 and mean work impact was 2.08 (on a scale from 1 to 5). $62.4 \%$ of subjects reported moderate satisfaction. Mental health workers with less schooling showed higher satisfaction. Work impact was not associated with any explanatory variable. The results for job satisfaction were similar to those of other studies. Work impact was very low. Unlike studies from the United States and Europe, there were no differences between the communitybased and in-hospital staff.

Mental Health; Health Personel; Job Satisfaction
Denise Rebouças 1

Lúcia Abelha 2

Letícia Fortes Legay 2

Giovanni Marcos Lovisi 2

\section{Introdução}

A partir de meados do século XX, nos países desenvolvidos, iniciou-se, de forma mais efetiva, o processo de reformulação da assistência em saúde mental, com a gradativa substituição do modelo centrado em hospitais por um tratamento de base comunitária 1,2. Na América Latina esse movimento aconteceu quase trinta anos depois, no processo da luta por direitos civis após décadas de ditaduras militares ${ }^{3}$.

Uma questão importante para a reforma psiquiátrica no Brasil, que já vem causando grande preocupação em países mais avançados em termos de desinstitucionalização, é lidar com aquela parcela da clientela que, mesmo sem os efeitos deletérios de longos períodos de institucionalização, apresenta transtornos graves e persistentes, desafiando as equipes mais instrumentalizadas pelos novos métodos e técnicas 4 . A precária situação econômica do Brasil, com sua desigualdade social crônica, não encoraja projeções otimistas quanto à criação de serviços comunitários na qualidade e quantidade necessárias. Entretanto, esse movimento vem progredindo, ainda que de forma lenta 5 .

A possibilidade de reprodução, nos serviços substitutivos, das características negativas do hospital psiquiátrico (descaracterização do indivíduo, institucionalismo, segregação, abandono), vem chamando a atenção de diversos autores, tornando absolutamente necessário o monito- 
ramento por meio da avaliação da qualidade da assistência ${ }^{6}$.

A avaliação em saúde mental apresenta dificuldades adicionais, já que envolve conceitos de difícil mensuração, como o bem-estar psíquico, sempre influenciado por fatores culturais e políticos 7,8 .

Os estudos de satisfação vêm sendo considerados importantes instrumentos de avaliação da qualidade em saúde mental por considerar a percepção dos segmentos envolvidos nos serviços 7,9.

Se na área de saúde o profissional é o principal instrumento de trabalho, em saúde mental esta afirmação é particularmente evidente 10,11. Sem as modernas tecnologias de exame e de tratamento constituídas por equipamentos e procedimentos cirúrgicos, o profissional e seus conhecimentos, adquiridos através da formação e da experiência, são os principais recursos com que se atua nesta área.

Os novos modelos de assistência em saúde mental envolvem a ampliação da oferta de cuidados aos pacientes, exigindo do profissional um envolvimento muito maior com a clientela, o que, no caso dos pacientes graves, pode prolongar-se por toda a vida. Essa nova forma de atuar pode acarretar uma sobrecarga para o profissional, constituindo-se, portanto, em potencial fator de influência sobre a qualidade da assistência.

A experiência internacional mostra a necessidade de investir no aumento da satisfação e de minimizar o impacto que o trabalho em saúde mental, especialmente nos serviços comunitários, traz aos profissionais. Diversos estudos apontam problemas decorrentes de insatisfação e sobrecarga dos profissionais, como o absenteísmo, a alta rotatividade, os altos níveis de estresse, gerando custos muito elevados e ameaçando a sustentabilidade desses serviços 12,13,14,15.

Embora os estudos de satisfação com a assistência em saúde mental no Brasil estejam sendo realizados com todos os segmentos envolvidos na assistência, a pesquisa dirigida à equipe técnica tem despertado menos interesse do que as que avaliam usuários e familiares, como se pode depreender pela quase inexistência de publicações a respeito ${ }^{16}$. Visando a contribuir para diminuir essa lacuna, o presente estudo teve como objetivo avaliar o nível de satisfação, aqui entendido como o sentimento resultante da interação de profissionais com suas crenças e valores e o ambiente de trabalho, e o impacto que este produz sobre a saúde e o bem-estar de profissionais lotados em serviços de saúde mental de uma área programática da cidade do Rio de Janeiro.

\section{Métodos}

\section{Delineamento do estudo}

Estudo com desenho seccional que avaliou diferentes tipos de serviços de atendimento em saúde mental.

\section{Local do estudo}

\section{- Hospital Municipal Jurandyr Manfredini}

Criado em 1983 como um dos serviços da antiga Colônia Juliano Moreira, para acolher a demanda de atendimento de pacientes externos, o hospital deveria servir para interromper o fluxo de pacientes considerados irrecuperáveis, que vinham de todo o país para permanecerem na instituição. O hospital, que pertencia à esfera federal, foi municipalizado e desvinculado da antiga estrutura em 1996 e tem sua atuação direcionada para a manutenção do paciente em seu ambiente social, dentro da política de saúde mental implantada nos hospitais psiquiátricos da cidade. Funciona como pólo de emergência da Área Programática 4 (AP-4), tem os únicos leitos psiquiátricos públicos disponíveis nesta área e oferece atendimento ambulatorial.

\section{- Serviços comunitários}

Devido ao funcionamento semelhante e ao pequeno número de profissionais lotados, os serviços Centro de Atenção Psicossocial (CAPS), Centro de Atenção Psicossocial Infantil (CAPSi) e o Programa Horto foram agrupados sob a denominação de "serviços comunitários".

\section{- Centro de Atenção Psicossocial (CAPS) Artur Bispo do Rosário}

Dispositivo integrante do Sistema Único de Saúde (SUS), de base comunitária, funciona em regime aberto e presta assistência a usuários com transtorno mental grave e persistente, devendo vir a substituir as internações hospitalares psiquiátricas 17.

A exemplo dos outros CAPS da cidade do Rio de Janeiro, o CAPS Artur Bispo do Rosário mantinha na época do estudo uma vinculação administrativa com o Hospital Municipal Jurandyr Manfredini. A assistência é multidisciplinar e funciona de segunda a sexta-feira, de $9 \mathrm{~h}$ às $17 \mathrm{~h}$. Os atendimentos podem ser grupais ou individuais e são oferecidas atividades organizadas em oficinas. O serviço dispõe também de programas de geração de renda, como o trabalho protegido na cantina do Hospital Municipal Jurandyr 
Manfredini e o Programa Horto. Na época do estudo 186 pessoas eram atendidas nesse CAPS.

\section{- Centro de Atenção Psicossocial (CAPSi) Eliza Santa Rosa}

Serviço de atenção diária, dedicado ao atendimento de crianças e adolescentes com transtorno mental grave. Atua de forma articulada com outros setores de assistência a essa população e, sempre que possível, com a família e quaisquer outras relações sociais do usuário em tratamento 17.

O CAPSi Eliza Santa Rosa foi inaugurado em 2001, com a transformação do ambulatório infanto-juvenil do Hospital Municipal Jurandyr Manfredini. Único CAPSi da AP-4, atua como ordenador técnico em assistência infanto-juvenil da área. Atende atualmente 230 pessoas. Funciona de segunda a sexta-feira, com equipe multidisciplinar, e os atendimentos podem ser: intensivo, semi-intensivo ou não intensivo. Três vezes por semana funciona a atividade chamada "porta de entrada". São atendidas todas as pessoas que procuram o serviço e oferecido encaminhamento adequado a cada situação, seja para a rede básica, para serviços de outras áreas ou para absorção no próprio CAPSi. Outras atividades desenvolvidas são: atendimento individual e grupal aos usuários, grupos de convivência, oficinas, atendimento e/ou acompanhamento de familiares, acompanhamento de usuários internados, acompanhamento de usuários em abrigos e escolas, reuniões de equipe e de supervisão. O CAPSi é responsável, ainda, pela supervisão clínica de uma residência terapêutica de adolescentes, administrada por uma Organização Nãogovernamental (ONG).

\section{- Programa Horto}

Programa de geração de renda do Hospital Municipal Jurandyr Manfredini, funciona em estreita vinculação com o CAPS Artur Bispo do Rosário. Quarenta e cinco usuários desenvolvem atividades de cultivo de plantas, culinária, costura, artesanato, venda dos produtos e venda de livros. Funciona de segunda a sexta-feira, com equipe multidisciplinar. O programa conta com parcerias na execução das atividades. O dinheiro arrecadado tem uma parcela reinvestida na compra de material, uma parte é retida para o funcionamento do serviço e o restante é dividido entre os usuários.

\section{População estudada}

Foram convidados a participar do estudo todos os profissionais que trabalhavam nesses serviços há, pelo menos, 12 meses, com exceção dos funcionários de empresas que executam serviços terceirizados. Os participantes do estudo eram profissionais das seguintes categorias: (a) nível superior: assistente social, enfermeiro, farmacêutico, médico, musicoterapeuta, psicólogo, terapeuta ocupacional; (b) nível médio: auxiliar de enfermagem, cuidador, técnico de enfermagem e funcionários que exercem funções administrativas ou de apoio. Existiam três tipos diferentes de vínculo profissional entre os servidores: federal, municipal e contratação por ONG. Alguns funcionários tinham mais de um vínculo de trabalho. Os diferentes vínculos profissionais apresentavam condições de trabalho diversas, em aspectos tais como salário, carga horária, estabilidade no emprego.

Os profissionais do estudo apresentavam algumas peculiaridades. Ao longo da história da instituição, as terras foram sendo ocupadas, primeiramente por servidores em casas funcionais, em seguida, por seus descendentes, que, em uma tradição informal, substituíam, também, os funcionários que se aposentavam nos postos de trabalho. Essa situação só se modificou no início da década de 80, com a implantação de equipes multidisciplinares, contratadas por meio de concurso público. Por essa razão, é possível encontrar até hoje funcionários que residem na área do imóvel da instituição, e que têm, portanto, vínculos outros com os pacientes. Podem ser encontrados, ainda, funcionários, sobretudo federais, cujos familiares também trabalham na instituição, constituindo esta comunidade uma rede particularmente complexa de relações.

\section{Coleta de dados}

O trabalho de campo foi realizado por quatro estagiários treinados, graduandos em psicologia, que percorreram as unidades distribuindo os questionários. Para garantir o anonimato, foi solicitado aos funcionários que não se identificassem no questionário e que, ao término do preenchimento, depositassem o material em uma urna. A coleta de dados foi precedida de reuniões nas unidades, apresentando a pesquisa e estimulando a participação dos servidores. Todos os turnos de trabalho foram cobertos pelos pesquisadores. Foi determinado um número de cinco retornos a cada unidade, com o objetivo de evitar a perda de respondentes. 
Instrumentos utilizados

- Escala de Avaliação da Satisfação da Equipe em Serviços de Saúde Mental (SATIS-BR abreviada)

É auto-administrada, contém 32 itens, cada um com respostas dispostas em escala Likert de 5 pontos, correspondendo a: (1) "muito insatisfeito"; (2) "insatisfeito"; (3) "indiferente"; (4) "satisfeito" e (5) "muito satisfeito". Os itens são agrupados em quatro fatores: (1) qualidade dos serviços oferecidos aos participantes; (2) participação da equipe no serviço; (3) condições de trabalho e (4) relacionamento no serviço. Além dos itens quantitativos, quatro questões qualitativas estão incluídas no questionário.

\section{- Escala de Avaliação do Impacto do Trabalho em Serviços de Saúde Mental (IMPACTO-BR)}

É auto-administrada, contém 18 itens, cada um com respostas dispostas em escala Likert de 5 pontos, correspondendo a: (1) "de forma alguma"; (2) "não muito"; (3) "mais ou menos"; (4) "muito" e (5) "extremamente". Os itens são agrupados em três fatores: (1) efeitos sobre a saúde física e mental; (2) efeitos no funcionamento da equipe e (3) repercussões emocionais do trabalho. Além dos itens quantitativos, estão incluídas três questões qualitativas no questionário.

São escalas desenvolvidas em projeto multicêntrico da Organização Mundial da Saúde (OMS), com estudo de validação para o Brasil realizado por Bandeira et al. 9 , com base em estudo canadense. As escalas globais apresentam elevada consistência interna e alta homogeneidade de itens, medidas usando-se o coeficiente alfa de Cronbach.

Juntamente com as escalas foi solicitado aos participantes que preenchessem um questionário sobre dados sócio-demográficos e situação profissional. As variáveis estudadas foram: sexo, idade, estado civil, tipo de vínculo profissional (municipal, federal ou funcionário de ONG), escolaridade, categoria funcional, local de trabalho e tempo de serviço.

\section{Análise dos dados}

Foram calculadas freqüências para variáveis categóricas e médias para variáveis contínuas. Para comparação de médias foram utilizados os testes estatísticos não paramétricos Mann-Whitney U e Kruskall Wallis. O teste qui-quadrado foi utilizado para medir associações entre variáveis. $\mathrm{O}$ teste de correlação de Pearson foi utilizado para medir correlação entre satisfação e impacto. Os resultados da análise bivariada foram considerados estatisticamente significativos quando o $\mathrm{p}$ valor foi igual ou menor que 0,05 . Foi realizada análise de regressão linear para examinar o efeito das variáveis do estudo. A estratégia utilizada foi a entrada de todas as variáveis que, na análise bivariada, apresentaram associação com p valor menor do que 0,20 . As variáveis com $p$ valor maior do que 0,20 foram sendo retiradas uma a uma, e os coeficientes comparados com os do modelo completo. Para diferenças maiores do que $10 \%$ foram testados interação e confundimento. O programa SPSS 10.1 (SPSS Inc., Chicago, Estados Unidos) foi utilizado para as análises estatísticas. Durante a análise dos dados, as categorias funcionais foram agrupadas por nível, superior ou médio, já que não foram observadas diferenças estatisticamente significativas nos níveis de satisfação e de impacto entre categorias dentro de cada um dos níveis.

O projeto foi apresentado e aprovado pelo Comitê de Ética em Pesquisa do Instituto Municipal Juliano Moreira e os participantes concordaram em assinar o termo de consentimento. $\mathrm{O}$ estudo foi conduzido de acordo com os princípios éticos contidos na Declaração de Helsinki e de suas emendas.

\section{Resultados}

Cento e trinta e três profissionais $(67 \%$ do total), sendo 105 no Hospital Municipal Jurandyr Manfredini e 28 nos serviços comunitários, participaram do estudo. Embora a perda de respondentes tenha sido maior do que seria desejável (33\%), não foram observadas diferenças significativas com relação aos participantes nas variáveis sócio-demográficas ou funcionais, com exceção dos psiquiatras que tiveram um percentual maior de não resposta.

Os participantes apresentavam as seguintes características: idade média: 43,8 anos, tempo médio de serviço: 117,6 meses. Os casados compunham a maioria $(56,8 \%)$, assim como as mulheres $(56,5 \%)$. Trinta e quatro vírgula um por cento exerciam cargo de nível superior. Sessenta e um vírgula um por cento eram servidores federais e o restante pertencia à esfera municipal. O nível de escolaridade distribuía-se da seguinte forma: $3 \%$ tinham ensino fundamental incompleto, $16,7 \%$ ensino fundamental completo, $35,6 \%$ ensino médio completo, $14,4 \%$ ensino superior completo e $30,3 \%$ pós-graduação.

O escore médio de satisfação global foi de 3,30 (DP = 0,66), com variação entre 1,25 e 4,84. A subescala que obteve a maior pontuação em 
satisfação foi a referente ao relacionamento no serviço: 3,81 ( $\mathrm{DP}=0,80$ ), variando entre 1,33 e 5,00 . Em seguida, qualidade dos serviços prestados aos pacientes: 3,45 ( $\mathrm{DP}=0,70)$, com variação entre 1,10 e 4,90. A terceira maior pontuação em satisfação foi obtida na subescala participação da equipe no serviço: 3,25 ( $\mathrm{DP}=0,89)$, variação de 1,00 a 5,00. As condições de trabalho constituem a subescala que gerou o nível mais baixo de satisfação: 3,05 (DP = 0,75), com o mínimo de 1,22 e o máximo de 4,67 . Seis vírgula oito por cento dos profissionais relataram estar insatisfeitos, $62,4 \%$ indiferentes e $30,8 \%$ satisfeitos.

Observou-se um escore médio de impacto global de 2,08 ( $\mathrm{DP}=0,79$ ), variando entre 1,00 e 5,00. As três subescalas variaram entre o mínimo de 1,00 e o máximo de 5,00. A subescala que contribuiu com o maior nível de impacto foi a referente às repercussões emocionais do trabalho: $2,34$ ( DP $=0,89)$. Em seguida, vem o impacto sobre o funcionamento da equipe, com média de $2,23$ ( $\mathrm{DP}=0,88)$. O impacto sobre a saúde física e mental foi a subescala que obteve o menor escore: $1,90(\mathrm{DP}=0,90)$.

Encontraram-se diferenças significativas nos escores de satisfação de acordo com o nível de escolaridade e com o nível da categoria funcional (Tabela 1). Após a análise por regressão, apenas o nível de escolaridade permaneceu associado ao nível de satisfação. Quanto mais alta a escolaridade, menor o nível de satisfação ( $\beta=-1,272$; $\mathrm{p}=$ 0,000; IC95\%: -1,949; -0,596).

$\mathrm{Na}$ análise bivariada foram encontradas associações do nível de impacto do trabalho com nível de escolaridade e nível da categoria funcional (Tabela 1). No entanto, após a análise por regressão não foram constatadas associações estatisticamente significativas.

Diferenças estatisticamente significativas nos níveis de satisfação e de impacto foram observadas na comparação entre os setores do hospital (Tabela 2) e na comparação entre os três serviços comunitários (Tabela 3).

\section{Discussão}

A generalização dos resultados deste estudo para outras equipes deve ser realizada com cuidado, uma vez que os serviços aqui estudados guardam peculiaridades relacionadas à sua origem (antiga Colônia Juliano Moreira).

Comparações de satisfação e sobrecarga de trabalho têm sido realizadas entre profissionais que atuam em serviços comunitários e enfermarias psiquiátricas em hospital geral, tendo sido encontrada uma gama variada de resultados 18,19,20. Em alguns desses estudos as equipes dos servi- ços comunitários apresentaram níveis mais altos de satisfação, mas também se mostraram mais sobrecarregadas com o trabalho do que os profissionais que atuam em hospitais.

Diferentemente desses estudos, nos serviços aqui investigados não foram encontradas diferenças estatisticamente significativas nos níveis de satisfação e impacto do trabalho entre profissionais que atuam em serviços comunitários e em ambiente hospitalar. Nos países desenvolvidos é possível observar com mais nitidez as diferenças entre os dois modelos de assistência. No Brasil, a instalação de serviços comunitários é recente, não sendo ainda tão evidentes as diferenças entre os serviços, o que pode ser uma explicação para os níveis de satisfação e de impacto semelhantes encontrados nos dois tipos de serviços. No caso particular deste estudo, os três serviços comunitários foram criados a partir do hospital e mantêm com este uma ligação administrativa e técnica, contribuindo para essa indiferenciação.

No entanto, quando se realiza uma comparação entre os setores do hospital, observa-se que a emergência e o ambulatório apresentam escores mais baixos de satisfação. Esses setores também apresentam os níveis mais altos de impacto. Esses são os locais do hospital em que os profissionais são submetidos a níveis mais elevados de tensão, uma vez que têm de tomar decisões de emergência e, muitas vezes, de forma solitária. Têm um contato intensivo com pacientes em crise e com quadro agudo. A quantidade de atendimentos, em função da quantidade reduzida de profissionais e da crescente demanda, expõe os profissionais a um trabalho que pode ser estressante.

A leitura das respostas das questões qualitativas revela que o suporte fornecido pelos colegas da equipe é o fator mais mencionado como fonte de satisfação e o que mais contribui para minimizar o impacto do trabalho. Esta pode ser a explicação para os resultados observados na emergência e no ambulatório, já que a atuação nestes setores costuma acontecer de forma mais individualizada, com menos troca entre os profissionais.

No caso dos serviços comunitários, a explicação para as diferenças observadas também pode ser o suporte oferecido pelos colegas, o que justificaria os melhores resultados de satisfação encontrados no CAPSi, que é o serviço mais antigo, com profissionais atuando juntos há mais tempo, o que faz supor uma equipe mais coesa.

Não foi possível encontrar explicações nas respostas das questões qualitativas para os melhores resultados de satisfação e impacto do trabalho encontrados entre os profissionais de nível médio, mas é possível deduzir que os servidores 
Escores médios de satisfação e impacto do trabalho, por nível de escolaridade e nível do cargo, entre os profissionais dos serviços estudados. Rio de Janeiro, Brasil, 2005

\begin{tabular}{|c|c|c|c|c|c|c|c|c|}
\hline & $\begin{array}{l}\text { Fundamental } \\
\text { incompleto } \\
\text { Média (DP) }\end{array}$ & $\begin{array}{l}\text { Fundamental } \\
\text { e médio } \\
\text { Média (DP) }\end{array}$ & $\begin{array}{l}\text { Superior } \\
\text { Média (DP) }\end{array}$ & $\begin{array}{c}\text { Pós- } \\
\text { graduação } \\
\text { Média (DP) }\end{array}$ & $\begin{array}{c}\chi^{2} \\
(\mathrm{df}=3)\end{array}$ & $\begin{array}{c}\text { Nível } \\
\text { médio } \\
\text { Média (DP) }\end{array}$ & $\begin{array}{c}\text { Nível } \\
\text { superior } \\
\text { Média (DP) }\end{array}$ & $Z$ \\
\hline Satisfação global & $4,40(0,17)$ & $3,36(0,57)$ & $3,10(0,75)$ & $3,14(0,68)$ & 14,69 * & $3,13(0,69)$ & $3,40(0,61)$ & $-2,19$ * \\
\hline \multicolumn{9}{|l|}{$\begin{array}{l}\text { 1. Qualidade dos } \\
\text { serviços oferecidos }\end{array}$} \\
\hline aos pacientes & $4,51(0,24)$ & $3,50(0,64)$ & $3,32(0,78)$ & $3,29(0,7)$ & 12,79 * & $3,29(0,73)$ & $3,55(0,67)$ & $-2,01 *$ \\
\hline \multicolumn{9}{|l|}{ 2. Participação da } \\
\hline equipe no serviço & $4,14(0,35)$ & $3,27(0,78)$ & $3,04(1,04)$ & $3,19(0,98)$ & 5,49 & $3,18(1,00)$ & $3,29(0,81)$ & $-0,58$ \\
\hline \multicolumn{9}{|l|}{ 3. Condições de } \\
\hline trabalho & $4,37(0,09)$ & $3,15(0,69)$ & $2,84(0,87)$ & $2,84(0,67)$ & 17,18 * & $2,82(0,69)$ & $3,20(0,75)$ & $-2,98$ * \\
\hline \multicolumn{9}{|l|}{ 4. Relacionamento } \\
\hline no serviço & $4,42(0,42)$ & $3,89(0,77)$ & $3,47(1,00)$ & $3,75(0,75)$ & 5,69 & $3,72(0,79)$ & $3,87(0,81)$ & $-1,17$ \\
\hline Impacto global & $1,40(0,53)$ & $2,07(0,88)$ & $1,95(0,65)$ & $2,08(0,79)$ & 7,42 & $2,20(0,69)$ & $2,00(0,85)$ & $-1,94$ * \\
\hline \multicolumn{9}{|c|}{ 1. Efeitos sobre a saúde } \\
\hline física e mental & $4,30(0,42)$ & $3,55(0,75)$ & $3,16(1,00)$ & $3,41(0,81)$ & 6,79 & $3,39(0,84)$ & $3,54(0,81)$ & $-0,97$ \\
\hline \multicolumn{9}{|l|}{ 2. Efeitos no } \\
\hline \multicolumn{9}{|l|}{ funcionamento da } \\
\hline equipe & $1,50(0,71)$ & $2,28(0,94)$ & $2,03(0,69)$ & $2,32(0,85)$ & 4,62 & $2,26(0,83)$ & $2,21(0,92)$ & $-0,51$ \\
\hline \multicolumn{9}{|l|}{ 3. Repercussões } \\
\hline emocionais & $1,35(0,7)$ & $2,21(0,94)$ & $2,37(0,85)$ & $2,65(0,72)$ & 12,93 * & $2,65(0,73)$ & $2,14(0,93)$ & $-3,54$ * \\
\hline
\end{tabular}

* $p<0,05$.

Tabela 2

Escores médios de satisfação e impacto do trabalho, por setor, entre os profissionais do Hospital Municipal Jurandyr Manfredini. Rio de Janeiro, Brasil, 2005.

\begin{tabular}{|c|c|c|c|c|c|c|c|}
\hline & $\begin{array}{l}\text { Hospital Municipal } \\
\text { Jurandyr Manfredini } \\
\text { Média (DP) }\end{array}$ & $\begin{array}{l}\text { Emergência } \\
\text { Média (DP) }\end{array}$ & $\begin{array}{l}\text { Enfermaria } \\
\text { Média (DP) }\end{array}$ & $\begin{array}{l}\text { Ambulatório } \\
\text { Média (DP) }\end{array}$ & $\begin{array}{l}\text { Adminis- } \\
\text { tração } \\
\text { Média (DP) }\end{array}$ & $\begin{array}{l}\text { Mais de um } \\
\text { Média (DP) }\end{array}$ & $\begin{array}{c}\chi^{2} \\
(\mathrm{df}=4)\end{array}$ \\
\hline Satisfação global & $3,26(0,68)$ & $2,92(0,74)$ & $3,44(0,52)$ & $2,96(0,54)$ & $3,61(0,56)$ & $2,98(0,75)$ & 17,04 * \\
\hline \multicolumn{8}{|l|}{ 1. Qualidade dos serviços } \\
\hline oferecidos aos pacientes & $3,41(0,72)$ & $3,14(0,81)$ & $3,57(0,54)$ & $3,16(0,59)$ & $3,69(0,62)$ & $3,05(0,89)$ & 10,93 * \\
\hline 2. Participação da equipe no serviço & $3,17(0,89)$ & $2,79(0,96)$ & $3,39(0,76)$ & $2,82(0,84)$ & $3,62(0,75)$ & $2,93(0,96)$ & 13,91 * \\
\hline 3. Condições de trabalho & $3,02(0,79)$ & $2,60(0,86)$ & $3,16(0,71)$ & $2,84(0,58)$ & $3,42(0,72)$ & $2,71(0,81)$ & 13,32 \\
\hline 4. Relacionamento no serviço & $3,85(0,83)$ & $3,64(0,92)$ & $4,05(0,56)$ & $3,49(0,90)$ & $4,07(0,73)$ & $3,92(0,80)$ & 6,73 \\
\hline Impacto global & $2,09(0,83)$ & $2,20(0,87)$ & $1,95(0,66)$ & $2,27(0,64)$ & $1,81(0,62)$ & $2,55(1,33)$ & 5,76 \\
\hline 1. Efeitos sobre a saúde física e mental & $1,92(0,97)$ & $1,89(1,05)$ & $1,71(0,71)$ & $2,21(0,76)$ & $1,67(0,71)$ & $2,43(1,60)$ & 5,48 \\
\hline 2. Efeitos no funcionamento da equipe & $2,27(0,93)$ & $2,43(0,88)$ & $2,17(0,82)$ & $2,28(0,81)$ & $2,03(0,80)$ & $2,74(1,37)$ & 3,23 \\
\hline 3. Repercussões emocionais & $2,32(0,88)$ & $2,51(0,87)$ & $2,22(0,75)$ & $2,65(0,59)$ & $1,92(0,80)$ & $2,73(1,20)$ & 9,20 \\
\hline
\end{tabular}

$* p<0,05$ 
Escores médios de satisfação e impacto do trabalho, por serviço, entre os profissionais dos serviços comunitários localizados no Complexo Juliano Moreira. Rio de Janeiro, Brasil, 2005

\begin{tabular}{|c|c|c|c|c|c|}
\hline & $\begin{array}{c}\text { Serviços } \\
\text { comunitários }\end{array}$ & CAPS & CAPSi & $\begin{array}{c}\text { Programa } \\
\text { Horto }\end{array}$ & $X^{2}(d f=4)$ \\
\hline & Média (DP) & Média (DP) & Média (DP) & Média (DP) & \\
\hline Satisfação global & $3,43(0,53)$ & $3,17(0,40)$ & $3,71(0,51)$ & $3,11(0,45)$ & 7,76 * \\
\hline 1. Qualidade dos serviços oferecidos aos pacientes & $3,58(0,62)$ & $3,32(0,56)$ & $3,90(0,54)$ & $3,14(0,53)$ & 8,03 * \\
\hline 2. Participação da equipe no serviço & $3,52(0,85)$ & $3,44(0,55)$ & $3,73(0,76)$ & $3,05(1,38)$ & 1,30 \\
\hline 3. Condições de trabalho & $3,18(0,56)$ & $2,74(0,41)$ & $3,50(0,53)$ & $3,09(0,32)$ & 10,28 * \\
\hline 4. Relacionamento no serviço & $3,69(0,70)$ & $3,78(0,44)$ & $3,76(0,80)$ & $3,33(0,78)$ & 1,37 \\
\hline Impacto global & $2,01(0,64)$ & $2,37(0,56)$ & $1,95(0,66)$ & $1,54(0,32)$ & 7,58 * \\
\hline 1. Efeitos sobre a saúde física e mental & $1,83(0,64)$ & $2,00(0,63)$ & $1,87(0,69)$ & $1,40(0,28)$ & 3,98 \\
\hline 2. Efeitos no funcionamento da equipe & $2,09(0,70)$ & $2,41(0,55)$ & $2,05(0,79)$ & $1,60(0,38)$ & 5,60 \\
\hline 3. Repercussões emocionais & $2,39(0,93)$ & $3,11(0,87)$ & $2,13(0,81)$ & $1,84(0,67)$ & 7,85 * \\
\hline
\end{tabular}

CAPS: Centro de Atenção Psicossocial; CAPSi: Centro de Atenção Psicossocial Infantil.

* $\mathrm{p}<0,05$.

mais qualificados tenham uma expectativa maior sobre o trabalho. O exame do nível de satisfação obtido para a pergunta sobre satisfação com o salário indica que os funcionários de nível médio são significativamente mais satisfeitos do que os de nível superior. Uma explicação plausível seria o fato de que, diferentemente do mercado, o serviço público não remunera os funcionários em consonância com sua qualificação. Para um profissional de nível superior do Ministério da Saúde ou da Secretaria Municipal de Saúde não há diferença, em termos de salário, entre os que têm e os que não têm pós-graduação. Na população estudada, a maioria (68\%) dos funcionários de nível superior tem pós-graduação.

Este estudo mostrou a importância de se realizar investimentos para elevar o nível de satisfação dos profissionais dos serviços de saúde mental. Embora a maior parte dos participantes do estudo apresente um nível intermediário de satisfação, este resultado pode indicar uma certa indiferença com o trabalho (note-se que a média global obtida foi de 3,30 e o item 3 de pontuação da SATIS-BR abreviada é "indiferente”). Esse achado é compatível com os resultados de um estudo de satisfação realizado com 175 profissionais de municípios brasileiros, em que a maioria apresentou escores bons ou regulares de satisfação ${ }^{21}$. Um problema crescente observado em outros países é a alta rotatividade entre os profissionais. Nos Estados Unidos há pesquisas mostrando que a cada cinco a sete anos muda toda a força de trabalho em saúde mental 14. Em nosso país pode-se imaginar que o desemprego e a escassa proteção social desencorajem mudanças de emprego, independente da satisfação e do estresse gerados pelo trabalho.

É importante considerar a possibilidade de que os profissionais menos satisfeitos e, sobretudo, os que sofrem com mais intensidade o impacto de trabalhar em saúde mental tenham sido excluídos do estudo por estarem afastados por licença médica. Questionários foram enviados, via correios, para essas pessoas, mas não houve retorno. O percentual de não resposta (33\%) pode ser devido à falta de tradição de participação dos profissionais no desenvolvimento de pesquisas como prática de rotina nos serviços, o que acarreta receios e descrença quanto à possibilidade de interferir no processo de trabalho 21 . $\mathrm{O}$ maior índice de não resposta encontrado entre os psiquiatras pode ser devido à concentração desta categoria nos setores de atendimento mais intensivo (emergência e ambulatório), o que explicaria sua menor disponibilidade em responder ao questionário.

O estudo evidenciou que os participantes mostravam desconforto em emitir opiniões a respeito de seu local de trabalho. Entretanto, é fundamental a implantação de mecanismos de avaliação sistemática dos serviços de saúde mental na comunidade, para a obtenção de dados com o objetivo de reorientação da assistência. É importante estimular o entendimento de que a avaliação é condição intrínseca para a realização de atividades de qualquer natureza 22,23. 


\section{Resumo}

Apesar da importância, no planejamento de políticas públicas, do conhecimento da satisfação e do impacto do trabalho nos profissionais de saúde mental, há escassez de estudos sobre o assunto no Brasil. Este estudo investigou a satisfação e o impacto do trabalho em 133 profissionais de quatro serviços de saúde mental do Rio de Janeiro, com as escalas SATIS-BR, IMPACTO-BR e um questionário sobre características sócio-demográficas e do trabalho. Para a análise das associações entre variáveis foram empregados os testes KruskalWallis, Mann-Whitney, qui-quadrado e regressão linear múltipla. O programa SPSS 10.1 foi utilizado nas análises. O escore médio de satisfação foi de 3,30 e o escore médio de impacto foi de 2,08, em escala variando de 1 até 5 . Sessenta e dois vírgula quatro por cento dos profissionais apresentaram um nível intermediário de satisfação. Observou-se que quanto maior a escolaridade, menor a satisfação. O impacto do trabalho não está associado com quaisquer das variáveis estudadas. O nível intermediário de satisfação obtido é compatível com os resultados de outros estudos. O impacto do trabalho sobre estes profissionais é reduzido. Diferentemente de outros estudos realizados nos Estados Unidos e Europa, não foram observadas diferenças entre equipes hospitalares e comunitárias.

Saúde Mental; Pessoal de Saúde; Satisfação no Emprego

\section{Colaboradores}

D. Rebouças coordenou o estudo, desde a concepção do projeto até a análise dos dados, e foi responsável pela redação do manuscrito. L. Abelha e L. F. Legay colaboraram em todas as etapas do estudo e fizeram a revisão do manuscrito. G. M. Lovisi contribuiu na revisão do manuscrito e nas análises estatísticas.

\section{Referências}

1. Lima LA, Lovisi G, Morgado A. Questões da bioética no contexto da reforma psiquiátrica. J Bras Psiquiatr 1999; 48:21-7.

2. Leff J, Trieman N. Long-stay patients discharged from psychiatric hospitals. Br J Psychiatry 2001; 176:217-23.

3. Vasconcelos EM. Avaliação de serviços no contexto da desinstitucionalização psiquiátrica: revisão de metodologias e estratégias de pesquisa. J Bras Psiquiatr 1995; 44:189-97.
4. Desviat M. Reforma psiquiátrica. Rio de Janeiro: Editora Fiocruz; 1999.

5. Abelha L, Legay LF, Lovisi G. O processo de reforma psiquiátrica brasileiro. Cad Saúde Coletiva 2004; 12:9-25.

6. Cavalcanti MT. Desafios e importância estratégica da avaliação em qualidade de serviços de saúde mental para a assistência psiquiátrica brasileira contemporânea. Cadernos do IPUB 1998; v. esp.:103-17. 
7. Pitta AM, Silva Filho JF, Souza GW, Lancman S, Kinoshita RT, Cavalcanti MT, et al. Determinantes da qualidade de serviços de saúde mental em municípios brasileiros: estudo da satisfação com os resultados das atividades desenvolvidas por pacientes, familiares e trabalhadores dos serviços. J Bras Psiquiatr 1995; 44:441-52.

8. Schmidt M. A avaliação da qualidade de serviços de saúde mental: uma contribuição para o campo da atenção psicossocial [Dissertação de Mestrado]. Rio de Janeiro: Instituto de Medicina Social, Universidade do Estado do Rio de Janeiro; 2003.

9. Bandeira M, Pitta AM, Mercier C. Escalas da OMS de avaliação da satisfação e da sobrecarga em serviços de saúde mental: qualidades psicométricas da versão brasileira. J Bras Psiquiatr 1999; 48:233-44.

10. Fonseca AC. A apuração de custos em serviço de saúde mental: o caso do S. Saúde Dr. Cândido Ferreira. In: Harari A, Valentini W, organizadores. A reforma psiquiátrica no cotidiano. São Paulo: Editora Hucitec; 2001. p. 25-36.

11. Libério M. Avaliação da qualidade de serviços de saúde mental. Saúde em Foco 2001; 21:61-77.

12. Pines A, Maslach C. Characteristics of staff burnout in mental health settings. Hosp Community Psychiatry 1978; 29: 233-7.

13. Wykes T, Stevens W, Everitt B. Stress in community care teams: will it affect the sustainability of community care? Soc Psychiatry Psychiatr Epidemiol 1997; 32:398-407.

14. Blankertz LE, Robinson SE. Turnover intentions of community mental health workers in psychosocial rehabilitation services. Community Ment Health J 1997; 33:517-29.
15. Carson J, Fagin L. Stress in mental health professionals: a cause for concern or an inevitable par of the job? Int J Soc Psychiatry 1996; 42:79-81.

16. Bandeira M, Pitta AM, Mercier C. Escalas brasileiras de avaliação da satisfação (SATIS-BR) e da sobrecarga (IMPACTO-BR) da equipe técnica em serviços de saúde mental. J Bras Psiquiatr 2000; 49:105-15.

17. Departamento de Ações Programáticas Estratégicas, Secretaria de Atenção à Saúde, Ministério da Saúde. Saúde mental no SUS: os Centros de Atenção Psicossocial. Brasília: Ministério da Saúde: 2004.

18. Prosser D, Johnson S, Kuipers E, Szmuckler G, Bebbington P, Thornicroft G. Mental health, "burnout" and job satisfaction among hospital and community-based mental health staff. Br J Psychiatry 1996; 169:334-7.

19. Reid Y, Johnson S, Morant N, Kuipers E, Szmukler G, Thornicroft G, et al. Explanations for stress and satisfaction in mental health professionals: a qualitative study. Soc Psychiatry Psychiatr Epidemiol 1999; 34:301-8.

20. Gigantesco A, Picardi A, Chiaia E, Balbi A, Morosini P. Job satisfaction among mental health professionals in Rome, Italy. Community Ment Health J 2003; 39:349-55.

21. Pitta AM. Qualidade de serviços de saúde mental: desafios para a epidemiologia. J Bras Psiquiatr 1996; 45:313-21.

22. Saraceno B, Asioli F, Tognoni G. Manual de saúde mental. São Paulo: Editora Hucitec; 1994.

23. Silva LMV, FormigliVLA. Avaliação em saúde:limites e perspectivas. Cad Saúde Pública 1994; 10:80-91.

Recebido em 19/Mar/2007

Versão final reapresentada em 14/Ago/2007

Aprovado em 30/Ago/2007 